\title{
General Anesthesia for Cesarean Section in a Pregnant Woman with Immune Thrombocytopenic Purpura (ITP): A Case Report and Review of the Literature
}

\section{Carlos Vera-Aguilera' ${ }^{1,3}$, Jose M. Torres-Zazueta ${ }^{1}$, Jena Konkler ${ }^{2}$, Jesus Vera-Aguilera ${ }^{2 *}$, Sergio Ariel Soto-Hopkins ${ }^{1,3}$}

${ }^{1}$ Facultad de Medicina, Universidad Nacional Autonoma de Mexico, Mexico

${ }^{2}$ Department of Hematology and Oncology, Essentia Health, Duluth, Minnesota, USA

${ }^{3}$ Departamento de Anestesiologis, Hospital de Especialidades, Centro Medico Nacional Siglo XXI, Mexico

Email: *jesusveraonc@gmail.com

How to cite this paper: Vera-Aguilera, C., Torres-Zazueta, J.M., Konkler, J., VeraAguilera, J. and Soto-Hopkins, S.A. (2022) General Anesthesia for Cesarean Section in a Pregnant Woman with Immune Thrombocytopenic Purpura (ITP): A Case Report and Review of the Literature. Open Journal of Anesthesiology, 12, 49-54.

https://doi.org/10.4236/ojanes.2022.121005

Received: December 1, 2021

Accepted: January 21, 2022

Published: January 24, 2022

Copyright $\odot 2022$ by author(s) and Scientific Research Publishing Inc. This work is licensed under the Creative Commons Attribution-NonCommercial International License (CC BY-NC 4.0). http://creativecommons.org/licenses/by-nc/4.0/ (c) (i) (3) Open Access

\begin{abstract}
Background: Management of immune thrombocytopenia (ITP) during pregnancy can be challenging, particularly by identifying a threshold for safe administration of neuraxial/general anesthesia and minimizing postpartum hemorrhage. There is controversy over the safety of cesarean section (CS) in ITP patients. In this case report, we discuss general anesthesia management in a patient with ITP with severe thrombocytopenia. Case Presentation: A 28-year-old female with relapsed/refractory ITP and severe thrombocytopenia underwent general anesthesia and emergent cesarean section with successful outcomes and minimal bleeding. Platelet counts before CS were 5000 $\times 10^{9} \mathrm{~L}$, the patient received 1 unit of platelets before the procedure and 1 unit of platelet and tranexamic acid $500 \mathrm{mg}$ was injected slowly during the procedure. No evidence of bleeding and no complications were observed in the patient or newborn. Conclusions: In an emergent circumstance, general anesthesia and cesarean section procedure were performed safely in a patient with severe thrombocytopenia, no hemorrhagic complications were seen for this patient or neonate. Objective of This Manuscript: To share our experience of a safe emergent CS procedure and general anesthesia in a patient with severe thrombocytopenia. Our experience may guide the management of ITP patients in emergent delivery circumstances.
\end{abstract}

\section{Keywords}

Immune Thrombocytopenia, Pregnancy, Cesarean Section, 


\section{Background}

Severe thrombocytopenia in pregnancy increases the risk of postpartum hemorrhage and neonatal complications [1]. Thrombocytopenia is a hematological condition of pregnancy with the most common type being pregnancy-associated thrombocytopenia (PAT) (65\% to $80 \%$ of the cases), followed by idiopathic thrombocytopenia (ITP), which is estimated to occur in 1 in 1000 to 1 in 10,000 pregnant women [1] [2].

ITP is characterized by increased platelet destruction by immunoglobulin G (IgG) antibodies, which theoretically can put the mother and fetus at high risk for hemorrhage. Anti-platelet IgG antibodies target the platelet membrane glycoproteins, fibrinogen receptor, glycoprotein Ib/IX and IIb/IIIa, Ia/IIa, V, and IV to induce their destruction in the reticuloendothelial system. ITP commonly presents with peripheral megakaryocytes and splenomegaly [3].

The platelet count decreases during pregnancy in all women beginning within the $1^{\text {st }}$ trimester, this finding may be attributed to physiological changes involving platelet dilution through increased plasma volume, hematic factors that ensue vascular modifications, morphological alterations, including increased spleen size that leads to further depuration of platelets within the low flow-rate circulation of the splenic sinusoids and a significant influence from placental circulation similar to that of the spleen circulation [4]. In comparison to non-pregnant women, pregnant women with ITP usually present with fewer bleeding symptoms, this is in part associated with the procoagulant state found in pregnancy attributable to increased levels of fibrinogen, factor VIII, and von Willebrand factor, with reduced activity of protein S [2].

Management of ITP in pregnancy requires a multidisciplinary approach in the peripartum to minimize neonatal and maternal bleeding. In general, treatment is initiated when symptoms occur (i.e. bleeding, petechia, wet purpura) or when the platelet count falls below $20-30 \times 10^{9} \mathrm{~L}$ [5]. If Cesarean Section (CS) is considered for delivery, the complications associated with the anesthetic technique should be considered, for example, locoregional techniques have a significant risk of neuraxial hematoma $(1: 168,000)$, obstetric general anesthesia on the other hand has the possibility of difficult airway access (1:224 - 390), increased hemorrhagic risk, delayed gastric emptying, risk of pulmonary aspiration and entails a mortality risk estimated at 6.5 per million [5].

In general, the lowest platelet count at which it is safe to administer spinal or epidural anesthesia remains controversial but generally, the recommendation is a platelet count of at least $75 \times 10^{9} \mathrm{~L}$ and at least $50 \times 10^{9} \mathrm{~L}$ is adequate for cesarean section [2] [6]. The decision about regional anesthesia is ideally made before delivery in conjunction with the obstetric anesthetist; each case must be individually considered, with the risk of the procedure (spinal hematoma) ba- 
lanced against benefits (pain relief, better blood pressure control, avoidance of general anesthesia) [6]. The American Society of Hematology recommends treatment for ITP if platelet counts are less than $30 \times 10^{9} \mathrm{~L}$ in the $2^{\text {nd }}$ and $3^{\text {rd }}$ trimester of pregnancy. First-line treatment options are intravenous immunoglobulin (anti-D) or corticosteroids (prednisone) with frequently reported adverse effects and moderate clinical response [7].

\section{Case Presentation}

A 28-year-old female gravida 1, para 0 was transferred to our hospital for ITP management during pregnancy. Her past medical history was relevant for ITP diagnosed 2 years before pregnancy (her ITP was refractory to multiple treatments including IVIG, Azathioprine, high doses of Dexamethasone, and finally achieved partial response to weekly Rituximab for 4 doses). At the time of pregnancy diagnosis, she was under observation with a platelet count of $\sim 160 \times$ $10^{9} \mathrm{~L}$.

The patient lost to follow up after her diagnosis of pregnancy because of the COVID-19 pandemic and presented to her local physician at week 29 of pregnancy with petechia in 4 extremities, no wet purpura was noted although her platelet count was $7 \times 10^{9} \mathrm{~L}$. At the time of presentation, she was also noted to have elevated blood pressure $150 / 80 \mathrm{mmHg}$, the patient was diagnosed with preeclampsia and started therapy with alfametildopa $500 \mathrm{mg}$ and hydralazine $50 \mathrm{mg}$ three times a day. Regarding her ITP, she was managed with $40 \mathrm{mg}$ of dexamethasone for 4 days, one dose of IVIG and one dose of Rituximab 375 $\mathrm{mg} / \mathrm{m}^{2}$ with no improvement of her platelets $\left(<30 \times 10^{9} \mathrm{~L}\right)$. In fact, at week 30 of pregnancy, her platelet count continued to decrease to $5 \times 10^{9} \mathrm{~L}$, there was also worsening petechia and vaginal bleeding, hemoglobin at the time was $6.5 \mathrm{~g} / \mathrm{dl}$ and the decision was to proceed with emergent CS at week 30.5 of gestation.

Prior to surgery, the patient's heart rate was 98 beats/min, regular, blood pressure $110 / 80 \mathrm{mmHg}$, and $\mathrm{SpO}_{2} 99 \%$. Bilateral normal vesicular breath sounds were heard on chest examination and other systems were normal. Continuous electrocardiogram, noninvasive $\mathrm{BP}$, and pulse oximetry monitoring were started. Pre-operative ultrasonography did not show evidence of fetal intracranial bleed. Standard monitoring was placed, two wide bore IV cannula were inserted, antiaspiration prophylaxis included metoclopramide $10 \mathrm{mg} / \mathrm{IV}$. After pre-oxygenating the patient for 10 minutes, anesthesia was induced with rapid sequence induction with fentanyl $3 \mathrm{mcg} / \mathrm{kg}$, rocuronium $1 \mathrm{mg} / \mathrm{kg}$ and propofol $1 \mathrm{mg} / \mathrm{kg}$ followed by oral intubation with King-Vision ${ }^{\circledR}$ video laryngoscope. Anesthesia was maintained with desflorane in 50\% oxygen and medical air. No subsequent doses of rocuronium were administered.

Platelets before CS were $5000 \times 10^{9} \mathrm{~L}$, there was concern among the team regarding platelet transfusion in patients with ITP based on conflicting evidence regarding its safety, the final consensus was to proceed with 1 unit of platelets before the procedure and 1 unit of platelet during surgery. Platelets after the procedure were $8 \times 10^{9} \mathrm{~L}$.Tranexamic acid $500 \mathrm{mg}$ was injected slowly during 
the procedure. A $2.9 \mathrm{~kg}$ baby was delivered within 5 minutes with an APGAR score of 8 and 10 at 1 and 5 minutes, respectively. Oxytocin $20 \mathrm{U}$ in $500 \mathrm{ml}$ of $0.9 \%$ saline was started followed by IV carbetocin $100 \mathrm{mcg}$. Intraoperative hemodynamics were stable. Total blood loss was $500 \mathrm{cc}$ during surgery, before extubating the patient sugammadex $200 \mathrm{mcg}$ was administrated. The patient was extubated on the table with no evidence of bleeding and no complications. Postoperative dexamethasone $40 \mathrm{mg}$ daily was started, and her platelet count slowly improved.

There was no neonatal thrombocytopenia or hemorrhagic complications. Post-operative pain was managed with ketorolac $30 \mathrm{mg}$ three times a day. Both mother and baby were discharged on day 6 . Upon discharge, platelet count was $55 \times 10^{9} \mathrm{~L}$ and hemoglobin of $9 \mathrm{~g} / \mathrm{dl}$, based on these findings, dexamethasone was discontinued. The platelet count of the newborn was $120 \times 10^{9} \mathrm{~L}$ on the first postnatal day. The patient and baby were evaluated at 3 weeks, both the mother and baby were in good health. Her platelet count was $>50,000 \times 10^{9} \mathrm{~L}$ with no treatment and the platelet count of the baby was $180 \times 10^{9} \mathrm{~L}$.

\section{Discussion}

Immune thrombocytopenia (ITP) is an acquired autoimmune disease caused by the destruction and impaired platelet production. In the general population, the incidence of ITP is estimated to be 2 to 5 per 100,000 persons [8]. In pregnancy, ITP is estimated to occur in 1 in 1000 to 1 in 10,000 women [5] [6]. The identification of ITP in pregnancy is challenging when solely based on the physiological decline of platelets; however, it is unlikely a gestational thrombocytopenia diagnosis if the platelet count is $<50 \times 10^{9} / \mathrm{L}$ [9].

The mode of delivery for pregnant patients with ITP is based on obstetric indications and concurrently avoiding procedures associated with an increased hemorrhagic risk to the fetus or the mother [9]. There is no consensus regarding the precise platelet count needed to safely perform neuraxial analgesia; however anesthesiologists will generally withhold spinal anesthesia for women with platelet counts $<75-80 \times 10^{9} \mathrm{~L}$ [9] [10] or $<50 \times 10^{9} \mathrm{~L}$ for a cesarean section [2] [3] [4] [5] [6]. Neonatal thrombocytopenia presented following almost $1 / 10$ of pregnancies with ITP. New pregnancy-onset disease was the only prognostic marker for neonatal thrombocytopenia [8].

Uncomplicated vaginal delivery in women with ITP was described in 1 case with platelet counts as low as $19 \times 10^{9} \mathrm{~L}$ and several other cases with platelet counts of $20-50 \times 10^{9} \mathrm{~L}$ [9] [10]. Nevertheless, the risk of conversion to $\mathrm{C}$-section is present in every labor and we agree with current recommendations to ideally aim for at least $50 \times 10^{9} \mathrm{~L}$ [9] [10].

In this patient, our team decided to proceed with general anesthesia to prevent neurological complications associated with regional anesthesia as her platelet count was $5 \times 10^{9} \mathrm{~L}$ and there was evidence of bleeding despite administration of IVIG, Rituximab, and high doses of steroids. Because of worsening bleeding and 
thrombocytopenia, the patient underwent emergent CS, 1 unit of platelet was transfused before surgery and 1 unit during CS.

Caution should also be taken while performing general anesthesia in patients with thrombocytopenia, as there is always the risk for traumatic injuries to the airway. In our patient, no evidence of bleeding or traumatic injury was identified. There is current controversy regarding platelet transfusion in ITP patients, in our patient no risk of thrombosis was identified with platelet transfusions.

In general, neuraxial blockade $(\mathrm{NAB})$ is preferred over general anesthesia as NAB provides reduced opioid adverse effects, as well as decreased mortality and morbidity; however, in patients with ITP undergoing NAB, caution should be taken as there is always a concern for hematoma or paraplegia [10]. Although there have been proponents of regional anesthesia in cases with severe thrombocytopenia, we report a case with general anesthesia based on the reasons above, without significant complications.

\section{Conclusion}

In an emergent situation, a cesarean section procedure performed in a patient with relapsed/refractory ITP and severe thrombocytopenia was safe and no neonatal thrombocytopenia or hemorrhagic complications were present.

\section{Acknowledgements}

The authors would like to acknowledge the Instituto Mexicano del Seguro Social for their support.

\section{Authors' Contributions}

All authors contributed equally to the development of this case. The authors read and approved the final manuscript.

\section{Ethics Approval and Consent to Participate}

This study was approved by the Ethics Committee of Instituto Mexicano del Seguro Social. The patient was assigned an informed written consent form.

\section{Consent for Publication}

Written informed consent was obtained from the patient for publication of this case report and any accompanying images. A copy of the written consent is available for review by the Editor-in-Chief of this journal.

\section{Conflicts of Interest}

The authors declare that they have no competing interests.

\section{References}

[1] Wang, X., Xu, Y., Luo, W., et al. (2017) Thrombocytopenia in Pregnancy with Different Diagnoses: Differential Clinical Features, Treatments, and Outcomes. Medicine 
(Baltimore), 96, Article No. e7561. https://doi.org/10.1097/MD.0000000000007561

[2] Provan, D., Stasi, R., Newland, A.C., et al. (2010) International Consensus Report on the Investigation and Management of Primary Immune Thrombocytopenia. Blood, 115, 168-186. https://doi.org/10.1182/blood-2009-06-225565

[3] Jodkowska, A., Martynowicz, H., Kaczmarek-Wdowiak, B. and Mazur, G. (2015) Thrombocytopenia in Pregnancy-Pathogenesis and Diagnostic Approach. Postępy Higieny i Medycyny Doświadczalnej (Online), 69, 1215-1221. https://doi.org/10.5604/17322693.1179649

[4] Reese, J.A., Peck, J.D., Deschamps, D.R., et al. (2018) Platelet Counts during Pregnancy. The New England Journal of Medicine, 379, 32-43.

https://doi.org/10.1056/NEJMoa1802897

[5] Amorim, J.G., Abecasis, M.R. and Rodrigues, F. (2018) Refractory Severe Thrombocytopenia during Pregnancy: How to Manage. Revista Brasileira de Ginecologia e Obstetrícia, 40, 803-807. https://doi.org/10.1055/s-0038-1675186

[6] Rolbin, S.H., Abbott, D., Musclow, E., Papsin, F., Lie, L.M. and Freedman, J. (1988) Epidural Anesthesia in Pregnant Patients with Low Platelet Counts. Obstetrics \& Gynecology, 71, 918-920.

[7] Eslick, R. and McLintock, C. (2020) Managing ITP, and Thrombocytopenia in Pregnancy. Platelets, 31, 300-306. https://doi.org/10.1080/09537104.2019.1640870

[8] Rottenstreich, A., Israeli, N., Roth, B., et al. (2020) Risk Factors Associated with Neonatal Thrombocytopenia in Pregnant Women with Immune Thrombocytopenic Purpura. Journal of Maternal-Fetal \& Neonatal Medicine, 33, 1572-1578. https://doi.org/10.1080/14767058.2018.1523891

[9] Gernsheimer, T., James, A. and Stasi, E. (2013) How I Treat Thrombocytopenia in Pregnancy. Blood, 121, 38-47. https://doi.org/10.1182/blood-2012-08-448944

[10] Choi, S. and Brull, R. (2009) Neuraxial Techniques in Obstetric and Non-Obstetric Patients with Common Bleeding Diatheses. Anesthesia \& Analgesia, 109, 648-660. https://doi.org/10.1213/ane.0b013e3181ac13d1 